\title{
On the nature of the hard X-ray source $4 U 2206+54$
}

\author{
I. Negueruela ${ }^{1,2,3,4}$ and P. Reig ${ }^{5,6}$ \\ 1 Observatoire de Strasbourg, 11 rue de l'Université, 67000 Strasbourg, France \\ 2 SAX SDC, Agenzia Spaziale Italiana, c/o Telespazio, via Corcolle 19, 00131 Rome, Italy \\ 3 Astrophysics Research Group, Liverpool John Moores University, Byrom St., Liverpool, L3 3AF, UK \\ 4 Physics and Astronomy Department, Southampton University, Southampton, SO17 1BJ, UK \\ 5 Foundation for Research and Technology-Hellas, 711 10, Heraklion, Crete, Greece \\ 6 Physics Department, University of Crete, 71003 Heraklion, Crete, Greece
}

Received 8 December 2000 /Accepted 26 March 2001

\begin{abstract}
The recent discovery of a $\sim 9.5 \mathrm{~d}$ period in the X-ray lightcurve of the massive X-ray binary $4 \mathrm{U} 2206+54$ has opened the possibility that it is a Be/X-ray binary with an unusually close orbit, which, together with its low intrinsic luminosity, suggests that the system is actually a Be + WD binary, in which a white dwarf accretes material from the dense circumstellar disc surrounding a classical Be star. In this paper we present new X-ray observations and for the first time high-resolution optical spectroscopy of the source. We show that both the X-ray behaviour and the characteristics of the optical counterpart, $\mathrm{BD}+53^{\circ} 2790$, are more consistent with a neutron star accreting from the wind of an early-type star. The X-ray lightcurve shows irregular flaring and no indications of pulsations, while the very high hydrogen column density supports accretion from a dense wind. BD $+53^{\circ} 2790$ is shown not to be a classical Be star, as believed until now, but rather a very peculiar late O-type active star, exhibiting emission components in the He II lines, complex spectral variability and strong wind resonance lines in the ultraviolet. Though many of the characteristics of the spectrum resemble those of the He-rich stars, the absence of He I variability makes a connection unlikely. The spectrum is compatible with a composite of two stars of similar spectral type, though circumstantial evidence points to a single very peculiar active early-type star. This adds weight to the growing evidence that the traditional subdivisions of supergiant and Be/X-ray binaries fail to cover the whole phenomenology of massive X-ray binaries.
\end{abstract}

Key words. stars: binaries: general - stars: early-type - X-rays: stars

\section{Introduction}

High Mass X-ray Binaries (HMXBs) are X-ray sources composed of an early-type massive star and an accreting compact object (generally a neutron star, but occasionally a black hole and, at least theoretically, possibly a white dwarf). HMXBs are traditionally divided (see Corbet 1986) into Classical or Supergiant X-ray binaries (SXBs) in which the compact object accretes from the stellar wind (sometimes directly from the atmosphere through localized Roche-lobe overflow) of an OB supergiant and $\mathrm{Be} / \mathrm{X}$-ray binaries (BeXBs), in which a neutron star orbits an unevolved OB star surrounded by a dense equatorial disc. Almost all known HMXBs fit well into one of these two categories (with a majority of systems being BeXBs), though a few systems, such as LMC X-4 (Hutchings et al. 1978) or RX J1826.2-1450 (Motch et al. 1997), seem to contain a compact object accreting from a "normal" main-sequence O-type star.

Send offprint requests to: I. Negueruela, e-mail: ignacio@astro.u-strasbg.fr
The hard X-ray source $4 \mathrm{U} 2206+54$ was first detected by the Uhuru satellite (Giacconi et al. 1972). It appeared in the Ariel $V$ catalogue as 3A 2206+543 (Warwick et al. 1981). Steiner et al. (1984; hereafter S84) used the refined position from the HEAO-1 Scanning Modulation Collimator to identify the optical counterpart with the early-type star $\mathrm{BD}+53^{\circ} 2790$. S 84 reported that the $\mathrm{H} \alpha$ line was in emission, showing two distinctly separated peaks with $\Delta v_{\text {peak }}=460 \mathrm{~km} \mathrm{~s}^{-1}$. From their photometry, they estimated that the counterpart was a B0-2e main sequence star, and therefore concluded that the system was a Be/X-ray binary. In this subclass of HMXBs, the X-ray emission is due to accretion of matter from a Be star by a compact companion (see Bildsten et al. 1997; Negueruela 1998). The name "Be star" is used as a general term describing an early-type luminosity class III-V star, which at some time has shown emission in the Balmer series lines (Slettebak 1988, for a review). Both the emission lines and the characteristic strong infrared excess when compared to normal stars of the same spectral types are attributed to the presence of circumstellar material in the 
shape of a decretion quasi-Keplerian disc (see Negueruela \& Okazaki 2000 for a recent discussion).

Assuming a distance to $4 \mathrm{U} 2206+54$ of $2.5 \mathrm{kpc}$, S84 calculate an average luminosity for the source of $L_{\mathrm{x}} \simeq 710^{34} \mathrm{erg} \mathrm{s}^{-1}$ between 1974 November and 1981 October. Saraswat \& Apparao (1992, henceforth SA92) presented X-ray observations of $4 \mathrm{U} 2206+54$ made with the EXOSAT satellite at different epochs between 1983-1985. The source was always detected, though in different states. In August 1983 and June 1985, the source was active, with a low-level luminosity of $\approx 510^{34} \mathrm{erg} \mathrm{s}^{-1}$ and aperiodic flaring phases (a few hundred seconds long) in which the overall X-ray flux increased by a factor $3-$ 5 and the X-ray spectrum changed, becoming harder. In December 1984, the source was in quiescence, and the Xray flux was weak $\left(L_{\mathrm{x}} \approx 310^{33} \mathrm{erg} \mathrm{s}^{-1}\right)$ and stable. SA92 also announced the possible detection of a spin period for the compact object which would be in the range $390-$ $400 \mathrm{~s}$ and suggested that the accreting object was a white dwarf.

The source appears in the ROSAT All Sky Survey (Voges et al. 1999) as 1RX J220755+543111 and has been consistently detected by the All Sky Monitor on board $R X T E$ according to the quick-look results provided by the $\mathrm{ASM} / R X T E$ team. Corbet et al. (2000) have announced the detection of a $9.570 \pm 0.004 \mathrm{~d}$ periodicity in the X-ray lightcurve. If this is the binary period, then it would be the shortest known for a BeXB - unless the $\sim 1.4 \mathrm{~d}$ periodicity in the optical lightcurve of RX J0050.7-7316 reflects its orbital period (Coe \& Orosz 2000).

\section{Observations}

$\mathrm{BD}+53^{\circ} 2790\left(=\mathrm{LS} \mathrm{III}+54^{\circ} 16=\right.$ Hilt 1086$)$ is included in several catalogues of bright stars. Measurements of its optical magnitudes are reported since the work of Hiltner \& Johnson (1956). In spite of this, very little previous work on this source has been reported. We have undertaken a major multi-wavelength monitoring campaign on this source, the results of which will be presented in a subsequent paper. Here we concentrate only on observations that provide information on the nature of the system.

\subsection{X-ray observations}

We have analysed X-ray data taken with the Proportional Counter Array (PCA) onboard the Rossi X-ray Timing Explorer $(R X T E)$. The data were retrieved from the $R X T E$ archive and correspond to an observation made on March 11-13, 1997. After the screening and filtering of data, i.e., ensuring that all five PCA units were functioning and removing data taken at low Earth elevation angle $\left(<10^{\circ}\right)$ and during times of high particle background, we were left with $\sim 9000 \mathrm{~s}$ of on-source clean data. Also in order to improve signal-to-noise we selected only events from the top layer (the PCUs have three Xenon layers, each consisting of two anode chains; see Jahoda et al. 1996 for a technical description of the instrument).

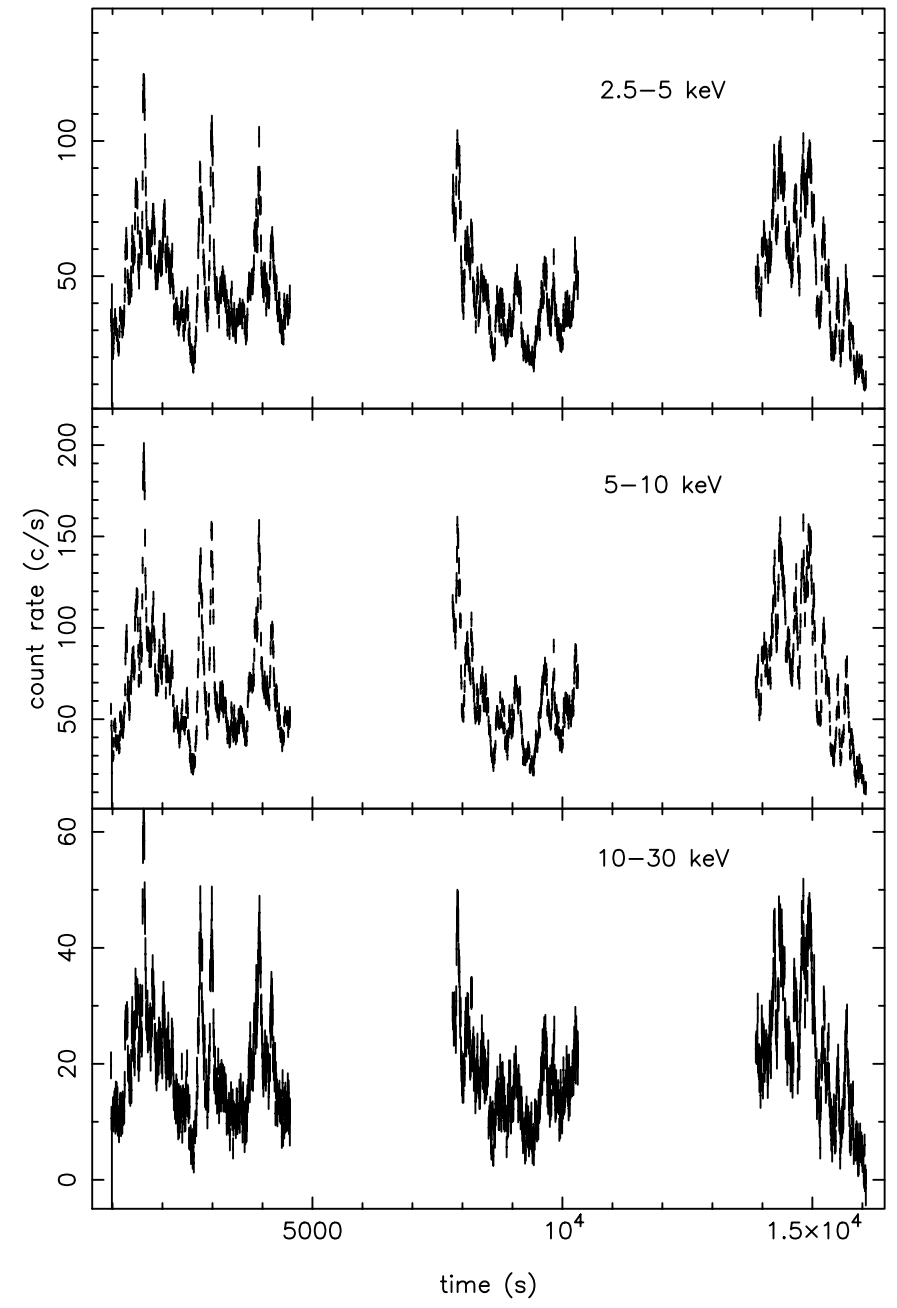

Fig. 1. Background subtracted RossiXTE lightcurves in three energy ranges. The original lightcurves have been rebinned into 8-s bins. The light-curve, clearly dominated by flaring activity, is similar to those of low X-ray luminosity supergiant binaries

Figure 1 shows the background subtracted light curves of $4 \mathrm{U} 2206+54$ in three different energy ranges. The temporal variability is characterised by erratic flaring activity on short timescales. The intensity shows changes by a factor of 3 in less than 2 min. The source becomes increasingly variable as the energy increases. The rms of the light curves varies from $\sim 40 \%$ for the energy range $2.5-5 \mathrm{keV}$ to $\sim 45 \%$ for $5-10 \mathrm{keV}$ and $\sim 50 \%$ for $10-30 \mathrm{keV}$. This flaring and erratic behaviour was also reported by SA92 during their observations (which covered the 2-10 keV range) on the two occasions in which the source was active. Similar lightcurves are observed in SXB binaries in which a neutron star accretes from the radiative wind of an evolved star, such as 2S 0114+65 (Yamauchi et al. 1990) or Vela X-1 (Kreykenbohm et al. 1999), which have typical $L_{\mathrm{x}} \sim 10^{35}-10^{36} \mathrm{erg} \mathrm{s}^{-1}$ (from now on, low-luminosity SXBs).

The correlation between the hardness ratio 5$10 \mathrm{keV} / 2.5-10 \mathrm{keV}$ and the count rate (see Fig. 2) indicates that the $\mathrm{X}$-ray spectrum becomes harder during the peak of the flares. 


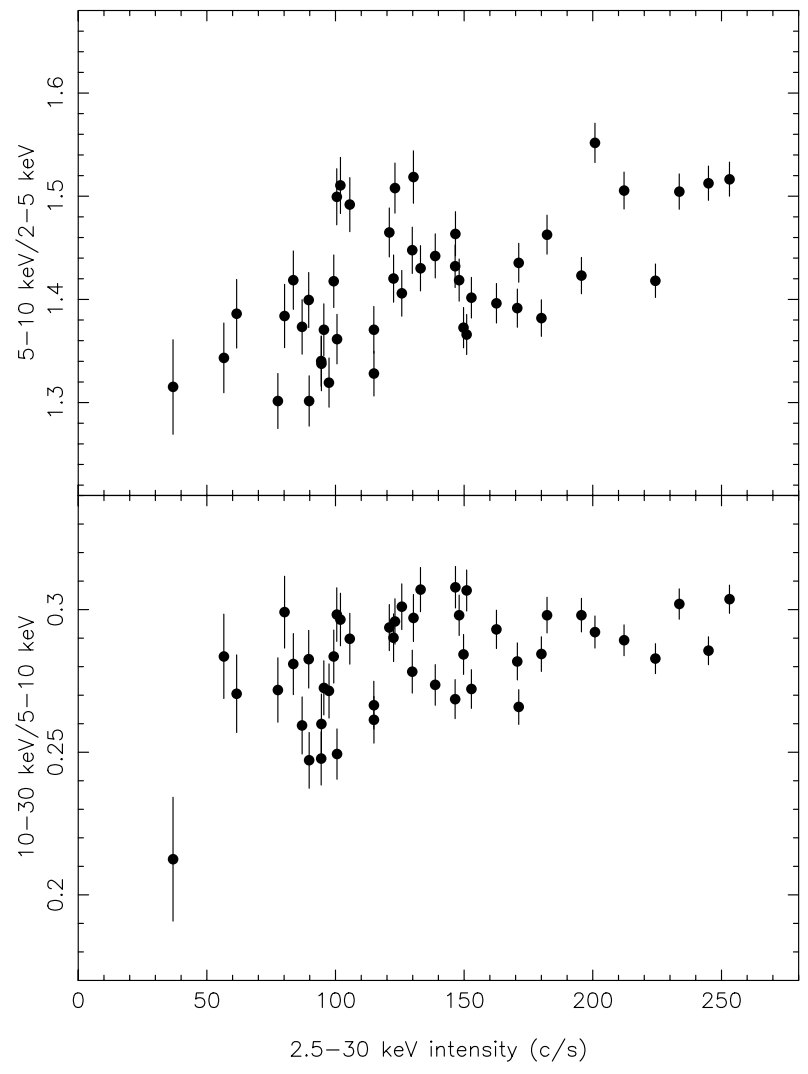

Fig. 2. Hardness ratios as a function of $2.5-30 \mathrm{keV}$ count rate. The X-ray spectrum tends to become harder as the count rate increases

Table 1. Spectral fit parameters and $68 \%$ confidence errors. The fit was performed in the energy range $2.5-30 \mathrm{keV}$

\begin{tabular}{lc}
\hline$N_{\mathrm{H}}\left(10^{22}\right.$ atoms cm & -2 \\
$\Gamma$ & $4.7 \pm 0.2$ \\
$E_{\text {cut }}(\mathrm{keV})$ & $1.71 \pm 0.3$ \\
$E_{\text {fold }}(\mathrm{keV})$ & $7.4 \pm 0.2$ \\
normalization & $17.5 \pm 0.8$ \\
$\chi_{\mathrm{r}}^{2}($ dof $)$ & $0.085 \pm 0.003$ \\
\hline
\end{tabular}

Figure 3 shows the power spectrum of $4 \mathrm{U} 2206+54$. No evidence for the $390-400 \mathrm{~s}$ pulse period reported by SA92 was found. The power spectrum is dominated by a strong red noise component and no periodicity is detected at any significative level.

Spectral analysis was performed on Standard2 data, in the energy range $2.5-30.0 \mathrm{keV}$. The best-fit model was an absorbed power-law and a high energy cutoff yielding an unabsorbed X-ray flux of $4.810^{-10} \mathrm{erg} \mathrm{cm}^{-2} \mathrm{~s}^{-1}$. The bestfit parameters and their $68 \%$ confidence errors are given in Table 1, while Fig. 4 shows the photon distribution. No evidence for an iron line at around $6.4 \mathrm{keV}$ was found. This line is seen in the spectra of the low-luminosity SXBs which display similar X-ray lightcurves. We can set an upper limit on the equivalent width of such line at $<10 \mathrm{eV}$.

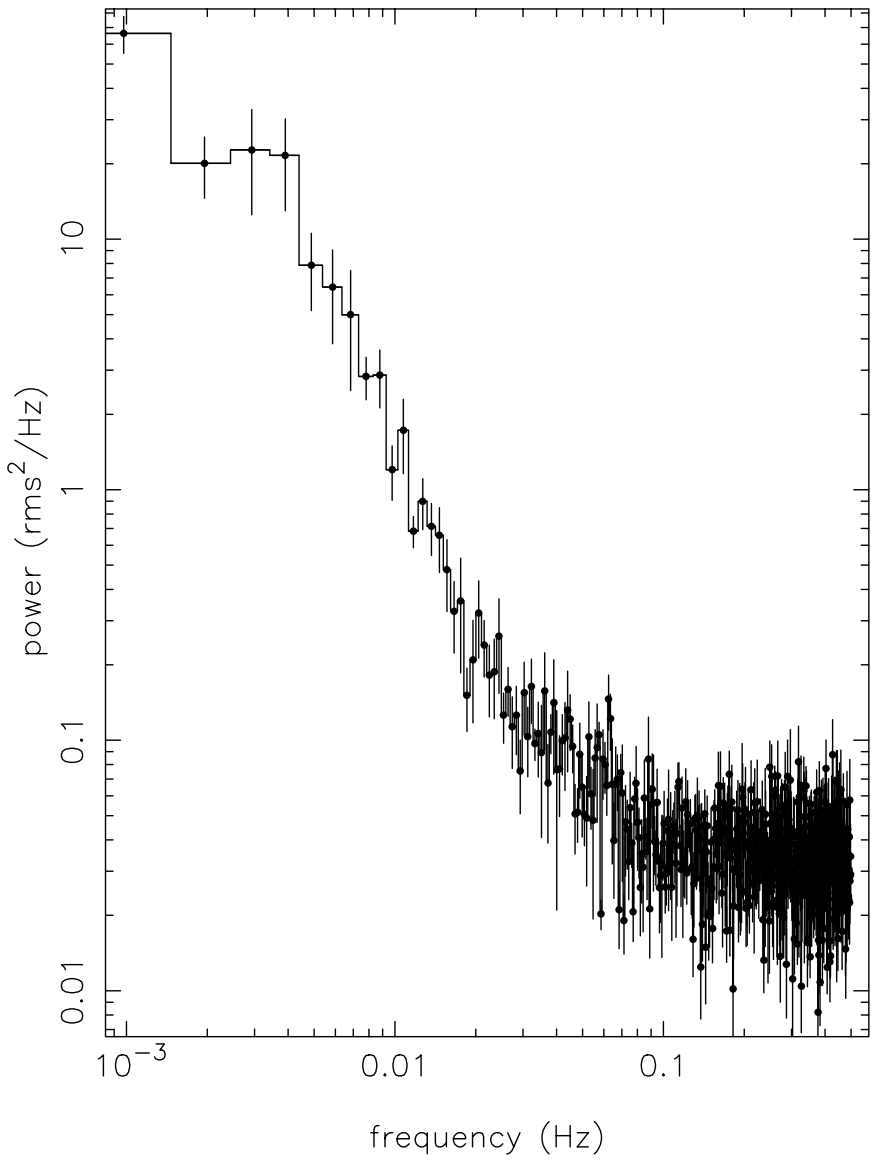

Fig. 3. Power spectrum for the $R X T E / \mathrm{PCA}$ observation of $4 \mathrm{U} 2206+54$

\subsection{Ultraviolet spectroscopy}

Ultraviolet observations of $\mathrm{BD}+53^{\circ} 2790$ were retrieved from the International Ultraviolet Explorer archive at Rutherford Appleton Laboratory. The database contains pre-processed spectra, which were subsequently reduced and analysed using the Starlink packages IUEDR (Giddins et al. 1996) and DIPSO (Howarth et al. 1997). The low resolution spectra from the Uniform Low Dispersion Archive (ULDA) LWP18128 and SWP39111 did not provide enough detail to allow accurate line identification. The high resolution spectrum SWP39112, taken with the short-wavelength camera in the large aperture mode on June 18, 1990, is displayed in Fig. 5. The original resolution of the spectrum is approximately $0.05 \AA$, but it has been rebinned to $0.4 \AA$ for display. The wavelength calibration, which has been checked with different interstellar lines, is accurate to a few $\mathrm{km} \mathrm{s}^{-1}$.

The most remarkable features are the strong P-Cygni profiles of the resonance wind doublets C IV $\lambda \lambda 1548.2$, $1550.8 \AA$ and $\mathrm{NV} \lambda \lambda 1239,1243 \AA$. They are stronger than those reported for Be stars of different spectral types by Prinja (1989) and resemble those typical of O-type main sequence stars (Walborn et al. 1985). The subordinate wind line NIV $\lambda 1718 \AA$, which generally follows the behaviour of the resonance doublets 


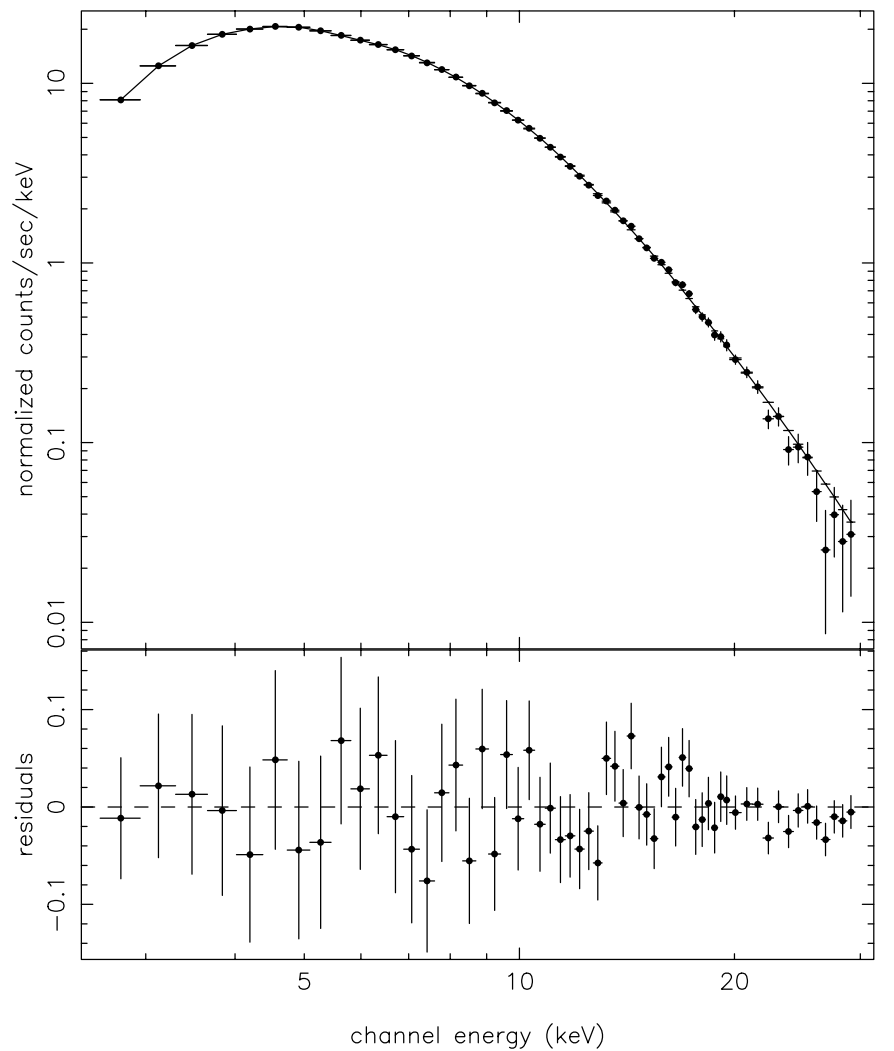

Fig. 4. X-ray spectrum of $4 \mathrm{U} 2206+54$ (circles) and the best-fit model (solid line). The continuum is represented by a powerlaw plus a cutoff at $7.4 \mathrm{keV}$ (see Table 1). No iron line is required

(Walborn \& Panek 1984a), does not show clear evidence for a P-Cygni profile, but it could be masked by the blend of two strong metallic lines just shortwards of it. The Si IV $\lambda \lambda 1394,1403 \AA$ doublet shows only a moderate wind effect, with narrow absorption troughs.

\subsection{Optical spectroscopy}

The source has been monitored since 1990, using a large array of telescopes and configurations. The complete dataset, its variability and searches for periodic behaviour will be reported in a forthcoming paper. Here we concentrate on relatively high-resolution blue spectroscopy taken with the 2.5-m Isaac Newton Telescope (INT), located at the Observatorio del Roque de los Muchachos, La Palma, Spain, on July 11, 1995 and August 3, 1998. Both observations were taken with the Intermediate Dispersion Spectrograph (IDS) equipped with the 235-mm camera and the R1200B grating. In 1995, the Tek3 CCD was in use, while in 1998, the EEV\#12 CCD had replaced it. Further blue observations were taken on July 2530, 2000, using the 1.52-m G. D. Cassini telescope at the Loiano Observatory, Italy, equipped with the Bologne Faint Object Spectrograph and Camera (BFOSC). Several observations were taken using grism \#6, while two higher resolution spectra were taken with grism\#9 in echelle mode (using grism\#10 as cross-disperser).
All the data have been reduced using the Starlink software packages CCDPACK (Draper 1998) and FIGARO (Shortridge et al. 1997) and analysed using DIPSO.

\section{The optical/UV spectrum}

The spectra of $\mathrm{BD}+53^{\circ} 2790$ in the classification region do not readily correspond to any spectral type. This fact was recognised by Hiltner \& Johnson (1956), who classified the star as O9IIIp from photographic plates (apparently not detecting any emission in the blue at the time). An immediate conclusion of our monitoring (see Fig. 6) is that the spectrum is also variable. The presence of strong He II lines, specially He II $\lambda 4200 \AA$, would indicate an O-type classification. Though the spectrum from August 1998 is relatively close to that of a normal O9 star with high $v \sin i$, most other blue spectra of the source (such as those from July 1995 and July 2000) display abundant and strong O II lines, together with a relatively strong Si III triplet. These lines do not correspond to an O-type star, but are typical of early B-type stars.

From our spectra, we can deduce the following information:

- He II $\lambda 4686 \AA$ is clearly variable, has obvious emission infilling and probably a permanent P-Cygni-like emission component.

- He II $\lambda 4200 \AA$ is possibly also variable, though not to the same extent as He II $\lambda 4686 \AA$.

- All O II and Si III lines are variable. They can be basically absent, as in the August 1998 spectrum, or rather strong, as in July 1995, with the July 2000 spectrum showing an intermediate state. Other metallic lines, such as N II $\lambda 4631 \AA$ and C II $\lambda 4267 \AA$ seem to participate in these changes, though N III $\lambda 4515 \AA$ apparently does not. All this would indicate a lower-temperature component superimposed on the spectrum of an $\mathrm{O} 9$ star.

- $\mathrm{H} \beta$, like $\mathrm{H} \alpha$ and the He I lines at $\lambda \lambda 6678,7065 \AA$ displays a shell-like spectrum, in the sense that the absorption feature is narrow and there are variable emission components in the wings. He I lines in the blue do not show this phenomenology.

- The upper Paschen series is basically in absorption, unlike in all the Be/X-ray binaries observed (see Negueruela \& Torrejón, in preparation).

The ultraviolet spectrum is typical of a main-sequence, late O-type star. The O-star classification is supported by the strength of the Fe V lines in the ultraviolet (specially the ratio Fe V $\lambda 1431 / \mathrm{C}$ III $\lambda 1427>1$; Walborn et al. 1985) and the strong He II $\lambda 4200 \AA$ and N III $\lambda 4515 \AA$ in the blue (Walborn \& Fitzpatrick 1990), none of which would be expected at the temperature of an early B-type star. The luminosity classification is supported by the shallow and broad photospheric lines in the ultraviolet and fundamentally the lack of wind troughs in the Si IV $\lambda \lambda 1394$, $1403 \AA$ resonance lines, which show a very strong luminosity dependence (Walborn \& Panek 1984b). 

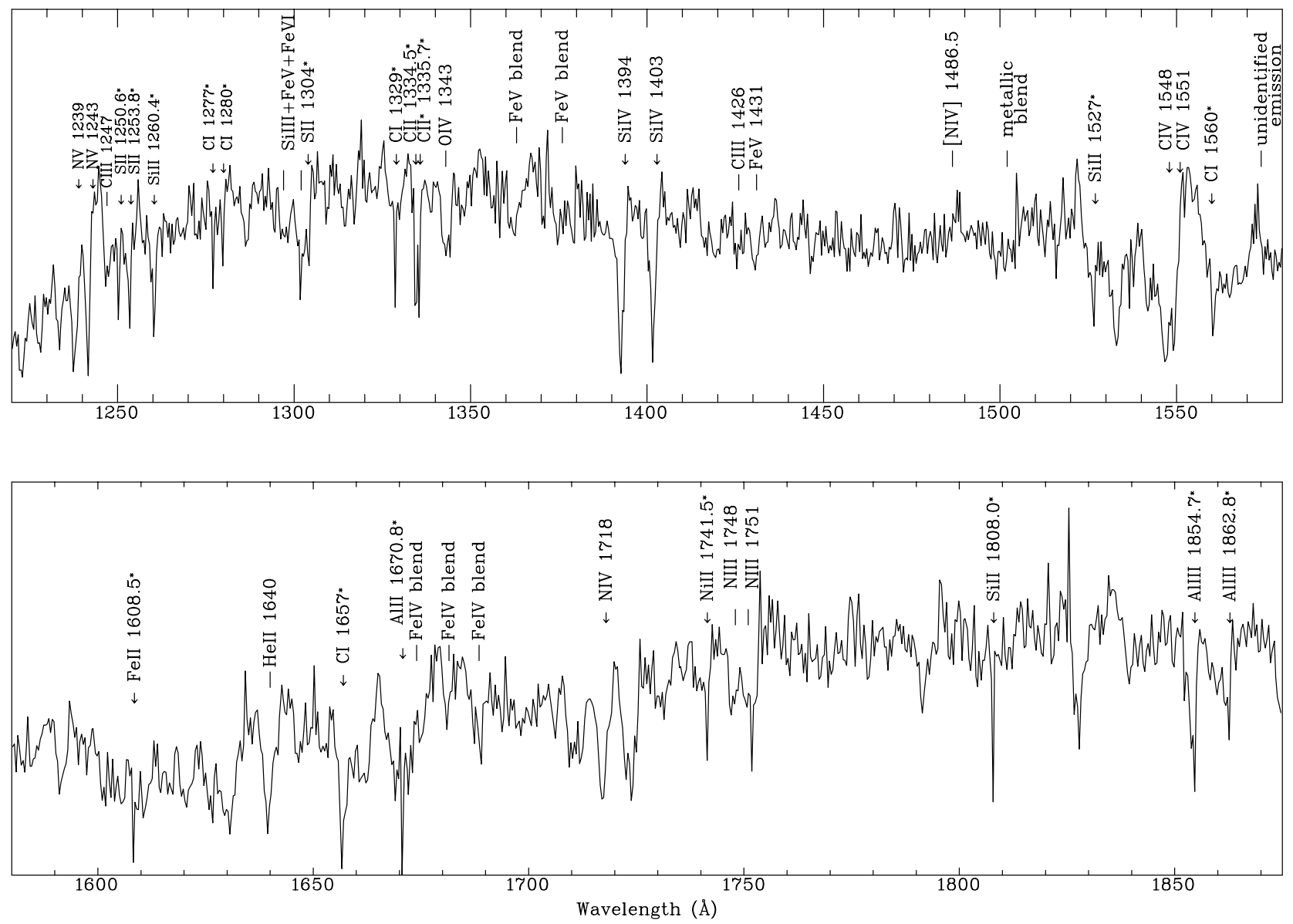

Fig. 5. The ultraviolet spectrum of $\mathrm{BD}+53^{\circ} 2790$. The arrows indicate the rest wavelength of the interstellar lines (marked with "**) and the wind lines N v $\lambda \lambda 1239,1243 \AA$, Si IV $\lambda \lambda 1393.8,1402.8 \AA$, C IV $\lambda \lambda 1548.2,1550.8 \AA$ and N IV $\lambda 1718 \AA$

The photospheric lines are those characteristic of a late-O/early-B main-sequence star, with $\mathrm{FeV}$ lines dominating the spectrum shortwards of $\lambda 1500 \AA$. The $\mathrm{Al}$ III $\lambda \lambda 1854.7,1862.8 \AA$ lines, although dominated by the sharp interstellar features seem to have shallow broader photospheric components, which would indicate a spectral type not much earlier than B0. The photospheric lines seem, in general, to be broader than those of the standards listed by Walborn et al. (1985).

In a normal star, the condition He II $\lambda 4545 \AA \simeq$ Si III $\lambda 4552 \AA$ would indicate a spectral type O9.5. The ratio between He I $\lambda 4471 \AA$ and He II $\lambda 4545 \AA$, which remains constant (He lines do not change intensity), also indicates a spectral type O9.5 (using the values from Mathys 1988). On the other hand, the presence of a variable spectral component, corresponding to a lower effective temperature, cannot be readily reconciled with the idea of a normal single O-type star. The following possibilities are open:

\section{1. $B D+53^{\circ} 2790$ as a peculiar Oe shell star}

The shapes of the $\mathrm{H} \alpha$ and HeI lines in the red are, at first sight, typical of a shell star. Shell lines are believed to be formed when the line of sight to the observer is intercepted by the outer cooler parts of the envelope of a Be star, which absorb the photospheric continuum (see Hanuschik 1995, 1996). Therefore Be stars seen very close to edge-on show deep absorption cores going down below the continuum level on top of their emission lines and are referred to as shell stars.

The spectra of Be shell stars are characterised by an absorption spectrum corresponding to a lower temperature (generally displaying many weak lines corresponding to Fe II and other singly-ionised metals) superimposed on the photospheric spectrum. To the best of our knowledge, the spectrum of an Oe shell star has never been described in the literature. It is therefore possible to speculate that the envelope surrounding an Oe shell star could produce the $\mathrm{O}$ II and $\mathrm{Si}$ III lines, which correspond to a temperature of $\sim 25000 \mathrm{~K}$.

This would not explain the emission component almost certainly seen in He II $\lambda 4686 \AA$ and would imply that either $\mathrm{BD}+53^{\circ} 2790$ is both an Of and an Oe star (the first ever identified) or that the He II emission originates in an accretion disc around the compact object. The presence of an accretion disc in the system, though not directly ruled out by observations, is difficult to reconcile with the absence of photometric variability. For example, Hiltner \& Johnson (1956) give $U=9.40, B=10.11, V=9.86$. 


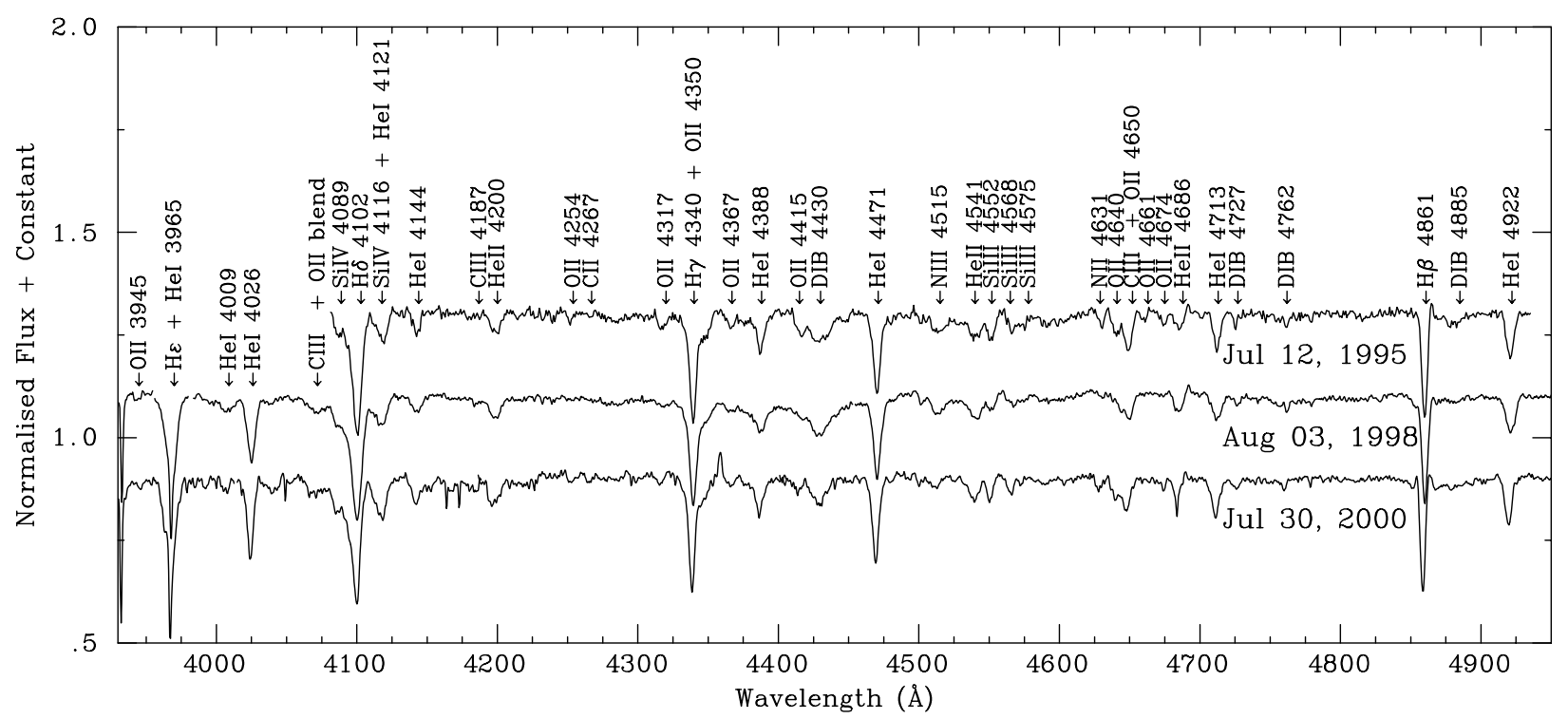

Fig. 6. High-resolution blue spectra of $\mathrm{BD}+53^{\circ} 2790$ showing spectral variability. All spectra have been divided by a spline fit to the continuum for normalization and offset by a constant amount. Diffuse Interstellar Bands (DIBs) are indicated

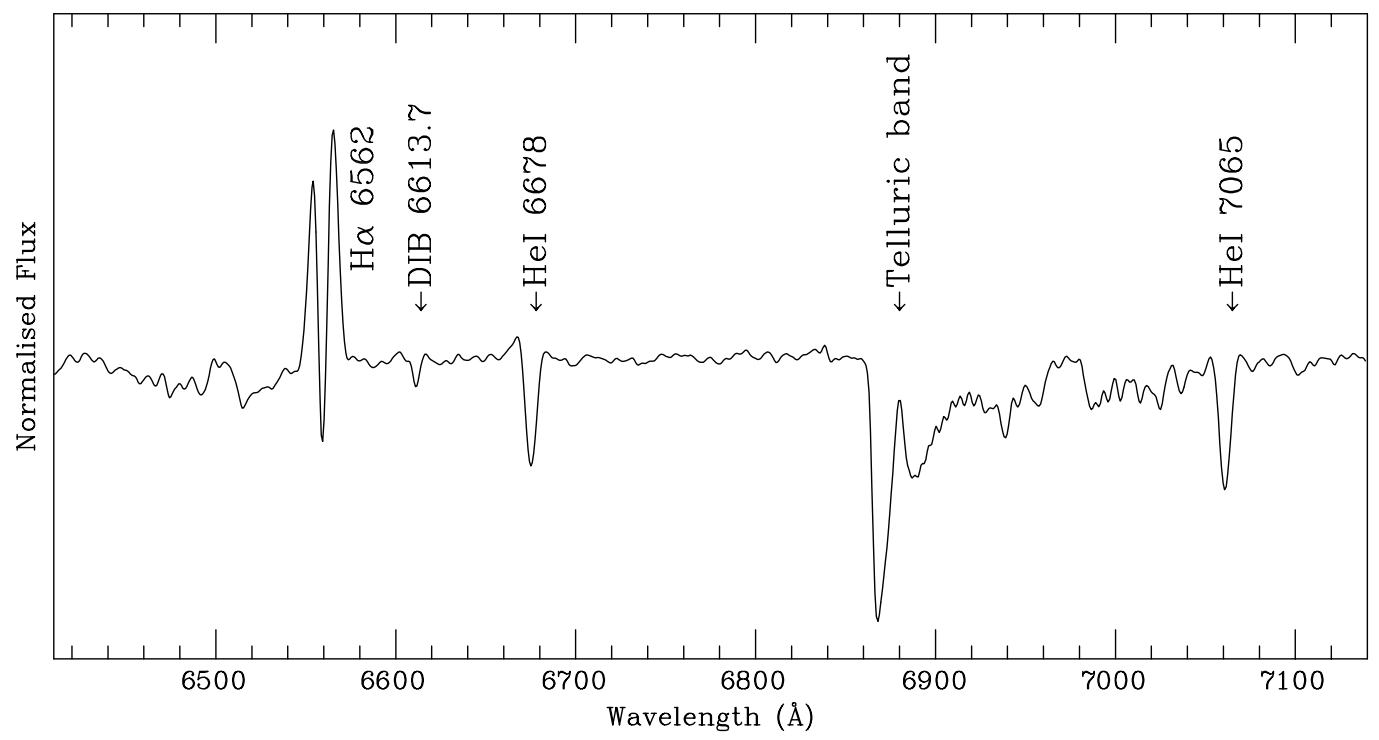

Fig. 7. Red spectrum of $\mathrm{BD}+53^{\circ} 2790$ (one order of the echelle spectrum taken on Jul. 29, 2000, from the 1.52-m G. D. Cassini Telescope) showing the typical characteristics of the spectra of the source in that region: shell-like features in H $\alpha$ and He I lines, $R>V$ asymmetry in $\mathrm{H} \alpha$ and the inverse symmetry in He I $\lambda 6678 \AA$. The spectrum has been divided by a polynomial fit to the continuum for normalisation and smoothed with a $\sigma=0.3 \AA$ Gaussian

Barbier et al. (1973) report $U=9.46, B=10.12$, $V=9.82$, while S84 give $U=9.45, B=10.05, V=9.85$, $R=9.61, I=9.41$ measured with the $0.91-\mathrm{m}$ telescope at Kitt Peak National Observatory on October 5, 1981. Given the expected errors (for instance, St84 estimate their errors at $0.04 \mathrm{mag}$ ), the values of $U B V$ are consistent with no variation at all. The Tycho catalogue gives magnitudes $B_{\mathrm{T}}=10.197 \pm 0.027$ and $V_{\mathrm{T}}=9.898 \pm 0.028$, which transforms into $B=10.13$ and $V=9.87$, again compatible with no changes at all.

A more obvious complication for this model is that the metallic lines do not seem to be any narrower than other presumably photospheric lines. As a matter of fact, it is difficult to estimate the rotational velocity of the star. Using the linear relationships for the width of He I lines developed by Steele et al. (1999) for Be stars observed with the same configuration, we obtain $v \sin i \approx 300 \pm 40 \mathrm{~km} \mathrm{~s}^{-1}$ from the lines on the August 1998 spectrum (i.e., that with the weakest metallic spectrum). This measurement clearly confirms that the star is a fast rotator, but there is a large dispersion in the values estimated from different lines.

Another problem faced by this interpretation comes from the asymmetry of the apparent shell lines. It is well known that in Be stars, symmetric lines arise from a quasi-Keplerian decretion disc, while asymmetric profiles correspond to perturbed configurations of such a disc 
(Hanuschik et al. 1988, 1995, 1996). Whenever asymmetric lines are seen, the ratio between the Violet and Red peaks ( $V / R$ ratio) varies quasi-cyclically, due to the development of global one-armed density oscillations (Okazaki 2000). In contrast, the $V / R$ ratio in the $\mathrm{H} \alpha$ line of $\mathrm{BD}+53^{\circ} 2790$ has been $<1$ during 15 years, in spite of the large changes in the overall shape and strength of the line. At the same time, the symmetry of $\mathrm{H} \beta$ and $\mathrm{H}$ I $\lambda 6678 \AA$ has changed. This behaviour has not been observed in other Be stars and makes it unlikely that $\mathrm{BD}+53^{\circ} 2790$ might be a classical Be star. Further arguments against the interpretation of $\mathrm{BD}+53^{\circ} 2790$ as a classical Be star will be discussed in a forthcoming paper.

One further complication is the detection of a likely $\approx 9.5 \mathrm{~d}$ period. It is difficult to see how an extended envelope could form with such a close binary companion. The only possibility would be that, due to tidal truncation by the companion (Negueruela \& Okazaki 2001), a very dense and small shell would form around the Oe star.

\section{2. $B D+53^{\circ} 2790$ as a spectroscopic binary}

One obvious possibility to explain the presence of a lowertemperature spectral component is that the star is in reality an unresolved spectroscopic binary, containing an O9 star and a slightly later companion. If this was the case, the companion should be of spectral type close to B1 in order to display the O II spectrum. Since the UV spectrum is clearly dominated by a main-sequence O-star, this companion cannot have a high luminosity and should be fainter than the O star in the UV and have a comparable magnitude in the $B$ band.

Such a combination could be obtained with, for example, an O9V((f))+B1III binary. The O9V star, due to its Of nature, would contribute the emission in He II and an asymmetric component to $\mathrm{H} \alpha$. The $\mathrm{B} 1$ giant could be a Be star and contribute the Balmer and He I line emission. It is clear that such configuration could never produce the observed X-ray luminosity because of colliding winds, since both stars would have relatively weak winds and even binaries with very strong winds produce lower luminosities. Moreover, the X-ray spectrum observed is very different from those of colliding wind systems, which are generally soft (with little signal above $10 \mathrm{KeV}$ ) and interpreted as coming from hot, optically thin plasma with fixed or variable solar element abundances (Skinner et al. 1998; Stevens et al. 1996). None of such models or a combination of them (MEKAL, VMEKAL, RAYMOND in XSPEC terminology) gave acceptable fits to the X-ray spectrum of $4 \mathrm{U} 2206+54$. Therefore, the presence of a compact object is necessary. Given the $\approx 9.5 \mathrm{~d}$ period, the compact object would be orbiting one of the components (presumably the Of star, since the Be star would not have a wind that could explain the observed X-ray lightcurve) in a close orbit and accreting from its wind. The other component would then be in a much wider orbit.
Abt \& Bautz (1963) found no evidence for binarity in $\mathrm{BD}+53^{\circ} 2790$ in their study of radial velocities of earlytype stars. The reported value $v_{\text {rad }}=-49.5 \mathrm{~km} \mathrm{~s}^{-1}$ is typical for a member of the Perseus arm. However, this does not rule out the binary model, since the system could be very wide. Accurate radial velocity measurements of the weak metallic lines in order to check whether they are consistent with the strongest lines, should be a test of this hypothesis.

\section{3. $B D+53^{\circ} 2790$ as a single peculiar active star}

The spectral variability of $\mathrm{BD}+53^{\circ} 2790$ resembles in many aspects that of He-rich stars, though these objects have later spectral types (clustered tightly around B2). Some of these systems are known to display spectral variability in their Si III lines in antiphase with their He I lines. These variations are strictly cyclical and correspond to the rotational period of the star, which is $\sim 1 \mathrm{~d}$ (Walborn 1982). Some of the He-rich stars (allegedly, those which are fast rotators) display $\mathrm{H} \alpha$ in emission, but the line profile does not look shell-like (Zboril et al. 1997). Only the peculiar He-rich star $\sigma$ Ori E displays an $\mathrm{H} \alpha$ line profile similar to that of $\mathrm{BD}+53^{\circ} 2790$, but in this star the $V / R$ ratio varies cyclically with the same period as the He I lines. In any case, $\mathrm{BD}+53^{\circ} 2790$ is unlikely to be related to He-rich stars (say, as an early-spectral type relative), because all the values of EWs measured for HeI lines in $\mathrm{BD}+53^{\circ} 2790$ are compatible with absolutely no changes in their strength or the $\mathrm{HeI} / \mathrm{H} \mathrm{I}$ ratio.

In spite of this, the possibility that the peculiar changes seen in the spectrum of $\mathrm{BD}+53^{\circ} 2790$ can be attributed to some unknown physical mechanism operating in a single star remains.

\section{Discussion}

Whatever the model adopted for $\mathrm{BD}+53^{\circ} 2790$, it is clear that its ultraviolet and blue spectrum is dominated by a star of approximate spectral type O9.5V. For such a star, the intrinsic colour would be close to $(B-V)_{0}=-0.3$. Then, using the value from St84, $(B-V)=0.2$, we have $E(B-V) \approx 0.5$ and, assuming standard reddening, $A_{V}=R \times E(B-V)=1.6$. An O9.5V star has an absolute magnitude $M_{V}=-4.3$ (Vacca et al. 1996) and therefore the measured $V=9.85$ implies $d \approx 3 \mathrm{kpc}$. At this distance, the average X-ray luminosity of $4 \mathrm{U} 2206+54$ is $L_{\mathrm{x}} \gtrsim 10^{35} \mathrm{erg} \mathrm{s}^{-1}$.

The observational history of $4 \mathrm{U} 2206+54$, which has been detected by all satellites that have pointed at it and has never been observed to undergo an outburst, is notably different from that of Be/X-ray transients, such as $4 \mathrm{U} 0115+63$ or A $0535+26$ (see Negueruela \& Okazaki 2000). There is a second subclass of Be/X-ray binaries characterised by low-luminosity, persistent X-ray emission with little variation, e.g., X Persei (Haberl et al. 1998), but they are believed to have large orbital periods (Reig \& Roche 1999) - which is certainly the case for 
X Persei (Delgado-Marti et al. 2001). The 9.5-d period of $4 \mathrm{U} 2206+54$ makes a connection unlikely, though the low X-ray luminosity Be/X-ray binary 3A 0726-26 $\left(P_{\mathrm{s}}=\right.$ $103.2 \mathrm{~s}$ ) could have a short period $P_{\text {orb }}=34.5 \mathrm{~d}$ (Corbet \& Peele 1997). Moreover, the decrease in $L_{\mathrm{x}}$ by more than one order of magnitude reported by SA92 is also atypical for these systems.

The X-ray luminosity and behaviour, with short erratic flares, point then to wind accretion as the mechanism producing the X-rays, if the compact object is a neutron star. The strong wind profiles seen in the UV spectrum indicate a large mass-loss rate which would fuel the X-ray system. Because of this, we can expect a similarity with low-luminosity SXBs, at least as far as the accretion process is concerned. Wind accreting supergiants with orbital periods similar to $4 \mathrm{U} 2206+54$ generally show rather higher luminosities. This is certainly the case of Vela X-1 $\left(P_{\mathrm{orb}}=8.96 \mathrm{~d}\right)$ with $L_{\mathrm{x}} \approx 410^{36} \mathrm{erg} \mathrm{s}^{-1}$ (Kreykenbohm et al. 1999) and likely $2 \mathrm{~S} 0114+65\left(P_{\text {orb }}=\right.$ $11.6 \mathrm{~d}$ ) if the large distance estimates are correct (e.g., Reig et al. 1996). Such differences would be due to the much weaker wind of the Of star compared to a supergiant.

In this respect, it must be noted that the absorption column to $4 \mathrm{U} 2206+54$ derived from our X-ray spectral fitting $N_{\mathrm{H}}=4.7 \pm 0.210^{22}$ atoms cm${ }^{-2}$ (not very different from the values found by SA92) is much larger than that corresponding to the interstellar absorption. The standard relation from Bohlin et al. (1978) indicates that $E(B-$ $V) \approx 0.5$ translates into $N_{\mathrm{H}} \approx 310^{21}$ atoms cm $\mathrm{cm}^{-2}$, i.e., one order of magnitude less than observed. This would indicate the presence of very optically thick material in the vicinity of the compact object, though it is not clear how this interpretation can be reconciled with the absence of an iron line.

It is also worth mentioning that the X-ray source RX $\mathrm{J} 1826.2-1450$, whose optical counterpart is also a mainsequence $\mathrm{O}$ star, could harbour a black hole, since it contains a microquasar (Paredes et al. 2000), and has an X-ray luminosity similar to or lower than $4 \mathrm{U} 2206+54$. Therefore we cannot rule out the possibility of a black hole companion in $4 \mathrm{U} 2206+54$, though the presence of a high-energy cutoff in the X-ray spectrum (which is typical of X-ray pulsars) favours a neutron star companion.

Even though we have no explanation for the spectral changes shown by $\mathrm{BD}+53^{\circ} 2790$, a very close similarity to $2 \mathrm{~S} 0114+65$ is suggested. The optical counterpart to this system, V662 Cas, apparently is a normal B1 supergiant (Reig et al. 1996), in spite of the fact that some authors have claimed that the strength of the Balmer lines is not as high as expected for such a star. However, van Kerkwijk \& Waters (1989) report an instance of spectral change in this source that occurred on November 4th, 1986. On this occasion, the complete metallic spectrum (i.e., O II + $\mathrm{Si}$ III) of the source - which is typical of a B1 supergiant disappeared, leaving behind what looked like a normal B23III spectrum. It is interesting that the same set of lines that are variable in $\mathrm{BD}+53^{\circ} 2790$ also varied in V662 Cas, though in the latter case, their disappearance seems to leave behind a cooler stellar spectrum. We take this as a suggestion that some stars in binaries with close compact object companions may be structurally unstable, perhaps due to their previous history, though at the moment we are unable to propose any physical mechanism for this variability.

Moreover Guarnieri et al. (1991) and Minarini et al. (1994) report the occurrence of optical outbursts in both $\mathrm{BD}+53^{\circ} 2790$ and V662 Cas, during which some lines which are generally not seen or in absorption go strongly into emission, and argue that these events suggest that both stars are Be stars. The classification spectra of both objects show that they are not classical Be stars, after all, but such episodes point to a further connection between the two systems. In this respect, it must be noted that Hall et al. (2000) have presented evidence strongly suggesting that the 2.7 -h periodicity observed in the $\mathrm{X}$-ray lightcurve of $2 \mathrm{~S} 0114+65$ should correspond to the spin period of a very slowly rotating neutron star. If $4 \mathrm{U} 2206+54$ exhibits periodic behaviour on a similar timescale, it is very unlikely that our observations could have detected it.

Whichever of the scenarios proposed turns out to be closer to reality, the X-ray emission from $4 \mathrm{U} 2206+54$ seems certain to be due to direct accretion from the wind of an active O-type star. This adds to increasing evidence that the traditional divisions of Supergiant X-ray binaries and $\mathrm{Be} / \mathrm{X}$-ray binaries are not enough to describe the whole set of Massive X-ray binaries. While the case of some objects, like 2S $0114+65$ and $4 \mathrm{U} 1907+09$, which seem to share characteristics of both groups, had always been shown to be problematic, it is clear now that there are a variety of objects, such as RX J1826.2-1450, RX J0050.7-7316 and 4U 2206+54, which simply do not belong to any of those categories.

\section{Conclusion}

We have presented X-ray observations of the High Mass Xray binary $4 \mathrm{U} 2206+54$. The erratic flaring, lack of pulsations and very high hydrogen column density strongly suggest that the X-ray emission is produced by accretion from a wind. Though circumstantial evidence supports the idea that the compact companion is a neutron star, the lack of pulsations and an X-ray luminosity comparable to that of RX J1826.2-1450, whose optical counterpart is a mainsequence star and contains a microquasar, leave the possibility of a black hole companion open. Optical and ultraviolet spectroscopy of the optical component BD $+53^{\circ} 2790$ show it to be a very peculiar object, displaying emission in $\mathrm{HI}$, HeI and He II lines and variability in the intensity of many metallic lines. Strong wind troughs in the UV resonance lines suggest a large mass loss rate. These properties might indicate that the star displays at the same time the Of and Oe phenomena or even hint at the possibility that it could be a spectroscopic binary consisting of two massive stars in addition to the compact object. There is with 
all certainty an $09.5 \mathrm{~V}$ star in the system, which is probably a mild Of star, and which likely feeds the compact object with its stellar wind.

Acknowledgements. The INT is operated on the island of La Palma by the Royal Greenwich Observatory in the Spanish Observatorio del Roque de Los Muchachos of the Instituto de Astrofísica de Canarias. The G. D. Cassini telescope is operated at the Loiano Observatory by the Osservatorio Astronomico di Bologna. This research has made use of data obtained through the High Energy Astrophysics Science Archive Research Center Online Service, provided by the NASA/Goddard Space Flight Center and of the Simbad data base, operated at CDS, Strasbourg, France.

We would like to thank the referee, Frank Haberl, for his helpful comments. IN would like to thank Prof. Nolan Walborn for useful discussions on the spectral classification and Dr. Manfred Pakull for valuable comments on the manuscript. The August 1998 spectrum was taken by Dr. I. A. Steele. During part of this work IN was supported by a PPARC fellowship and later by an ESA external fellowship. PR acknowledges support from the European Union through the Training and Mobility of Researchers Network Grant ERBFMRX/CT98/0195.

\section{References}

Abt, H. A., \& Bautz, L. P. 1963, ApJ, 138, 1002

Barbier, M., Bernard, A., Bigay, J. H., \& Garnier, R. 1973, A\&A, 27, 421

Bildsten, L., Chakrabarty, D., Chiu, J., et al. 1997, ApJS, 113, 367

Bohlin, R. C., Savage, B. D., \& Drake, J. F. 1978, ApJ, 224, 132

Coe, M. J., \& Orosz, J. A. 2000, MNRAS, 311, 169

Corbet, R. H. D. 1986, MNRAS, 220, 1047

Corbet, R. H. D., \& Peele, A. G. 1997, ApJ, 489, L83

Corbet, R. H. D., Remillard, R., \& Peele, A. G. 2000, IAUC, 7446

Delgado-Marti, H., Levine, A. M., Pfahl, E., \& Rappaport, S. A. 2001, ApJ, 546, 455

Draper, P. W. 1998, Starlink User Note 139.7, R.A.L.

Giacconi, R., Murray, S., Gursky, H., et al. 1972, ApJ, 178, 281

Giddings, J., Rees, P., Mills, D., \& Clayton, M. 1996, Starlink User Note 37.11, R.A.L.

Guarnieri, A., Bartolini, R., Civello, R., et al. 1991, in Structure and emission properties of accretion disks, ed. C. Bertout, et al. (Éditions Frontières, Gif sur Yvette), 435

Haberl, F., Angelini, L., Motch, C., \& White, N. E. 1998, A\&A, 330,189

Hall, T. A., Finley, J. P., Corbet, R. H. D., \& Thomas, R. C. 2000, ApJ, 536, 450

Hanuschik, R. W. 1995, A\&A, 295, 423

Hanuschik, R. W. 1996, A\&A, 308, 170

Hanuschik, R. W., Kozok, J. R., \& Kaiser, D. 1988, A\&A, 189, 147

Hanuschik, R. W., Hummel, W., Dietle, O., \& Sutorius, E. 1995, A\&A, 300, 163

Hanuschik, R. W., Hummel, W., Sutorius, E., et al. 1996, A\&AS, 116, 309

Hiltner, W. A., \& Johnson, H. L. 1956, ApJ, 124, 367

Howarth, I., Murray, J., Mills, D., \& Berry, D. S. 1997, Starlink User Note 50.20, R.A.L.
Hutchings, J. B., Crampton, D., \& Cowley, A. P. 1978, ApJ, 225,548

Jahoda, K., Swank, J. H., Stark, M. J., et al. 1996, in EUV, Xray and Gamma-ray Instrumentation for Space Astronomy VII, ed. O. H. W. Siegmund, \& M. A. Gummin, SPIE 2808, 59

van Kerkwijk, M. H., \& Waters, L. B. F. M. 1989, in 23rd ESLAB Symp. on Two Topics in X-ray Astronomy, Bologna, Italy 13-20 September 1989, ESA SP-296, Nov. 1989, 473

Kreykenbohm, I., Kretschmar, P., Wilms, J., et al. 1999, A\&A, 341,141

Mathys, G. 1988, A\&AS, 76, 427

Minarini, R., Teodorani, M., Bartolini, C., et al. 1994, in The Evolution of X-Ray Binaries, ed. S. S. Holt, \& C. S. Day, AIP Conf. Proc. 308, 275

Motch, C., Haberl, F., Dennerl, K., Pakull, M. W., \& Janot-Pacheco, E. 1997, A\&A, 323, 853

Negueruela, I. 1998, A\&A, 338, 505

Negueruela, I., \& Okazaki, A. T. 2000, in IAU Colloq. 175, The Be Phenomenon in Early-Type Stars. San Francisco, ed. M. Smith, H. F. Henrichs, \& J. Fabregat, ASP Conf. Ser. 214, 713

Negueruela, I., \& Okazaki, A. T. 2001, A\&A, 369, 108

Okazaki, A. T. 2000, in IAU Colloq. 175, The Be Phenomenon in Early-Type Stars. San Francisco, ed. M. Smith, H. F. Henrichs, \& J. Fabregat, ASP Conf. Ser. 214, 409

Paredes, J. M., Martí, J., Ribó, M., \& Massi, M. 2000, Science, 288,2340

Prinja, R. K. 1989, MNRAS, 241, 721

Reig, P., Chakrabarty, D., Coe, M. J., et al. 1996, A\&A, 311, 879

Reig, P., \& Roche, P. 1999, MNRAS, 306, 100

Saraswat, P., \& Apparao, K. M. V. 1992, ApJ, 401, 678 (SA92)

Shortridge, K., Meyerdicks, H., Currie, M., et al. 1997, Starlink User Note 86.15, R.A.L

Slettebak, A. 1988, PASP, 100, 770

Skinner, S. L., Masayuki, I., \& Nagase, F. 1998, New Astron., 3,37

Steele, I. A., Negueruela, I., \& Clark, J. S. 1999, A\&AS, 137, 147

Steiner, J. E., Ferrara, A., Garcia, M., et al. 1984, ApJ, 280, $688(\mathrm{~S} 84)$

Stevens, I. R., Corcoran, M. F., Willis, A. J., et al. 1996, MNRAS, 283, 589

Vacca, W. D., Garmany, C. D., \& Shull, J. M. 1996, ApJ, 460, 914

Voges, W., Aschenbach, B., Boller, Th., et al. 1999, A\&A, 349, 389

Walborn, N. R. 1982, PASP, 94, 322

Walborn, N. R., \& Fitzpatrick, E. L. 1990, PASP, 102, 379

Walborn, N. R., \& Panek, R. J. 1984a, ApJ, 286, 718

Walborn, N. R., \& Panek, R. J. 1984b, ApJ, 280, L27

Walborn, N. R., Nichols-Bohlin, J., \& Panek, R. J. 1985, IUE Atlas of O-Type Spectra from 1200 to $1900 \AA$ A, NASA Reference Publication 1155

Warwick, R. S., Marshall, N., Fraser, G. W., et al. 1981, MNRAS, 197, 865

Yamauchi, S., Asaoka, I., Kawada, M., Koyama, K., \& Tawara, Y. 1990, PASJ, 42, L53

Zboril, M., North, P., Glagolevskij, Yu. V., \& Betrix, F. 1997, A\&A, 324, 949 